\title{
Variación espacial de variantes fenotípicas en tropas de llamas productoras de fibra del Norte de la Provincia de Jujuy, Argentina
}

\author{
Michel Víctor Hubert Hick ${ }^{1} \bowtie$ (D) Alejandro Prieto, María Flavia Castillo, Eduardo Frank² (iD \\ Facultad de Ciencias Agropecuarias, Universidad Católica de Córdoba, Argentina
}

\section{Spatial variation on phenotypic variants of fiber production lama herds from Northern Jujuy Province, Argentine}

\begin{abstract}
In the north of the Province of Jujuy (Argentina), the lama population retains a great variability of its ethnozootechnical characteristics. Based on the scheme on the evolution of post-domestication populations, it has been stated that this variability determines a generalized situation of primariness, although there is an incipient standardization process. The objective was to choose the phenotypic variants (VFe) of the ethnozootechnical characters that explained the standardization process in preliminary work and to carry out a geostatistical analysis that allow to understand the spatial variation in the population of domestic camelids (llamas) to be studied. Georeferenced information from 85 troops from the North of the Province of Jujuy carried out between 1991 and 2008 was used. The chosen VFe were full fleece cover morphotype, white spotting design, absent/present staple raw pigmentation, luster staple types, using the respective relative frequencies (FRe, \%) calculated by herd. The greater presence of animals with $\mathrm{VFe}$, that is, higher $\mathrm{FRe}$, are indicators of selection and standardization. A geostatistical analysis consisted of the transformation of the geographic coordinates was carried out, obtaining an empirical semivariogram, evaluating the degree of spatial structuring and adjusting different semivariogram models and using kriging interpolation, obtaining a spatial variability map (MVs) for each phenotypic variant. Geostatistical analysis was performed from the exponential model which presented the best and highest values of spatial structuring. The MVs of these VFe showed an important spatial variation indicating an important generalized primariness with low values of FRe and on the other hand they confirm the existence of a well-defined area in the central and upper zone (Cieneguillas) with high values of FRe and a process of standardization. Through the geostatistical analysis, the spatial variation presented by the studied lama herds could efficiently and clearly be observed.
\end{abstract}

Key words: Camelids, variability, georeferencing, primariness, standardization.

Resumen. En el Norte de la Provincia de Jujuy (Argentina), la población de llamas conserva una gran variabilidad de sus características etnozootécnicas. En base al esquema sobre la evolución de las poblaciones pos-domesticación, se ha enunciado que dicha variabilidad determina una situación generalizada de primariedad, aunque existe un proceso de estandarización incipiente. El objetivo consistió en escoger las variantes fenotípicas (VFe) de los caracteres etnozootécnicos que explicaron el proceso de estandarización en trabajos preliminares y realizar un análisis geoestadístico que permita comprender la variación espacial en la población de Camélidos domésticos (llamas) a estudiar. Se utilizó información georreferenciada de 85 tropas del Norte de la Prov. de Jujuy realizado entre 1991 y 2008. Las VFe escogidas fueron morfotipo calzado, diseño de mancha total, pigmentación de mecha crudo o ausente y tipos de mechas lustres, utilizando las respectivas frecuencias relativas (FRe, \%) calculadas por tropa. La mayor presencia de animales con dichas VFe es decir mayor FRe, son indicadoras de selección y estandarización. Se realizó un análisis geoestadístico el cual consistió en la transformación de las coordenadas geográficas, obtención de un semivariograma empírico, evaluación del grado de estructuración espacial y ajuste de diferentes modelos de semivariograma y por interpolación kriging, obtención de un mapa de variabilidad espacial (MVs) para cada variante fenotípica. Se realizó el análisis geoestadístico a partir del modelo exponencial el cual presentó los mejores y elevados valores de estructuración espacial. Los MVs de dichas VFe mostraron una importante variación espacial indicando una importante primariedad generalizada con valores bajos de FRe y por otra parte confirman la existencia de un área bien delimitada en la parte central y superior (Cieneguillas) con valores elevados de FRe y un proceso de estandarización. Mediante el análisis geoestadístico se pudo observar de manera eficiente y clara la variación espacial que presentan las tropas de llamas estudiadas.

Palabras clave: Camélidos, variabilidad, georreferenciación, primariedad, estandarización,

Recibido: 2020-07-24. Aceptado: 2020-10-10

${ }^{1}$ Autor para la correspondencia: michelhicko9@gmail.com 


\section{Introducción}

En la Provincia de Jujuy existe una población de Camélidos domésticos de 123670 llamas (87.5 \% de la población nacional) criados por 2145 productores y en su mayoría se encuentran en el norte de la provincia, en la Puna. (INDEC, 2002; SENASA, 2012). Dicha región puneña está conformada por un altiplano árido por encima de los 3200 m.s.n.m y los Camélidos domésticos constituyen un importante recurso zoogenético autóctono y son la base de los sistemas productivos. En la década de los '9o se inició en dicha región un amplio relevamiento de tropas en base a caracteres etnozootécnicos. Los estudios para las primeras cuencas de producción en el altiplano jujeño ya indicaron que la población conserva una gran variabilidad de sus características etnozootécnicas como morfología, fenotipo de color y el producto fibra (Frank et al., 1996; Frank et al., 1999 y Frank, 2001). Luego ello fue indicado para una importante cantidad de tropas de llamas en numerosas cuencas de producción de la puna jujeña en los departamentos de Santa Catalina, Rinconada, Cochinoca y Yavi (Hick et al., 2009; Hick et al., 2014).

Lauvergne (1996) y Renieri et al. (2009) proponen un enfoque etnozootécnico para el entendimiento de la variabilidad que pueden presentar poblaciones domésticas. Dicho enfoque se basa en la evolución de las poblaciones pos-domesticación y diferentes estados en el que se pueden encontrar, desde un estado de primariedad general hasta diferentes estados de estandarización. Además, Lauvergne (1996) propone un esquema complementario desde el punto de vista geográfico para la comprensión de la evolución de una población pos-domesticación. El estado de primariedad se caracteriza por la gran variabilidad sobre todo de caracteres etnozootécnicos del tipo morfológico y visibles de los individuos de las tropas y de forma generalizada a lo largo de toda una región. Luego la evolución de una población puede dar a lugar al paso por diferentes estados de estandarización $\mathrm{u}$ homogeneización mediante la aparición primero de "puntos calientes" o islas de estandarización y luego su extensión hasta la formación de un mosaico de razas o biotipos estandarizados (razas propiamente dichas o en el sentido clásico). El inicio del proceso de estandarización consiste generalmente en la selección a favor de determinadas variantes fenotípicas dentro de un grupo de caracteres etnozootécnicos. Dichos autores junto a Hick (2015) aplicaron dicho enfoque etnozootécnico a la población de Camélidos domésticos, posibilitando una mejor comprensión de la variabilidad que exhiben dichas poblaciones. Paralelamente Tixier-Boichard et al. (2007), incorporado luego por FAO (2012), también proponen un similar abordaje para toda población doméstica y Hick et al. (2019) realiza una revisión y aplicación de los esquemas para los Camélidos Sudamericanos domésticos.

En base a los enfoques etnozootécnicos enunciados y producto de un relevamiento amplio y de numerosas tropas de la población del norte de la Provincia de Jujuy, se determinó una situación generalizada de primariedad para toda la población relevada. Además, se pudo observar tropas de un área denominada Cieneguillas, con un proceso de estandarización en principio insipiente. Ello se determinó mediante la utilización de índices de primariedad o arcaísmo confeccionados para las tropas relevadas a partir de caracteres etnozootécnicos y las variantes fenotípicas presentes. Dichos índices de primariedad fueron analizados con técnicas estadísticas multivariadas convencionales (Hick, 2015; Hick et al., 2018a).

La geoestadística aporta herramientas de análisis que permiten explorar y analizar la variabilidad espacial de variables regionalizadas (Oliver and Webster, 2015; Córdoba, 2015). A partir de información georreferenciada o regionalizada de las tropas y con estrategias de análisis de datos espaciales se podría profundizar los estudios realizados previamente. Por ello preliminarmente se realizó un primer análisis de la variabilidad espacial de la población de llamas del Norte de la Provincia de Jujuy en base al estudio de la primariedad o arcaísmo medida con un índice (Hick et al., 2018b). Los índices de primariedad se calcularon en base a numerosas variantes fenotípicas de caracteres etnozootécnicos que describen una población de llamas como los referidos a la morfología, al fenotipo de color de la capa y otras características de la capa asociadas con la calidad de la fibra como el tipo de mecha y la pigmentación de la mecha. Recientemente Hick et al. (2019) y de manera preliminar proponen realizar análisis geoestadísticos univariados a partir de determinadas variantes fenotípicas.

En el presente trabajo se propuso como objetivo escoger las variantes fenotípicas de los caracteres etnozootécnicos que explicaron el proceso de estandarización en trabajos preliminares y realizar un análisis geoestadístico que permita comprender la variación espacial en tropas de Camélidos domésticos (llamas) productoras de fibra en el norte de la Provincia de Jujuy. 


\section{Materiales y Métodos}

Se utilizó el relevamiento sistematizado e informado por Hick (2015) y Hick et al. (2018a) para tropas de diferentes cuencas de producción en el Norte de la Provincia de Jujuy realizado entre 1991 y 2008. En dicho estudio se relevaron 10973 llamas de un total de 17022 pertenecientes a 173 tropas y 9 cuencas de producción de los departamentos de Santa Catalina, Rinconada, Cochinoca y Yavi. El relevamiento fue llevado a cabo mediante la metodología demográfica de estructura poblacional descripta por Hick et al.
(2019). Para el análisis geoestadístico se utilizó la información georreferenciada (coordenadas geográficas) disponible y recuperada de 85 tropas de dicho relevamiento y empleadas previamente por Hick et al. (2018b) y Hick et al. (2019). Dichas tropas representan casi el $50 \%$ de las tropas originalmente relevadas y en la Figura 1 se observa su distribución dentro de las 9 cuencas de producción del relevamiento presentado por Hick (2015) y Hick et al. (2018a).

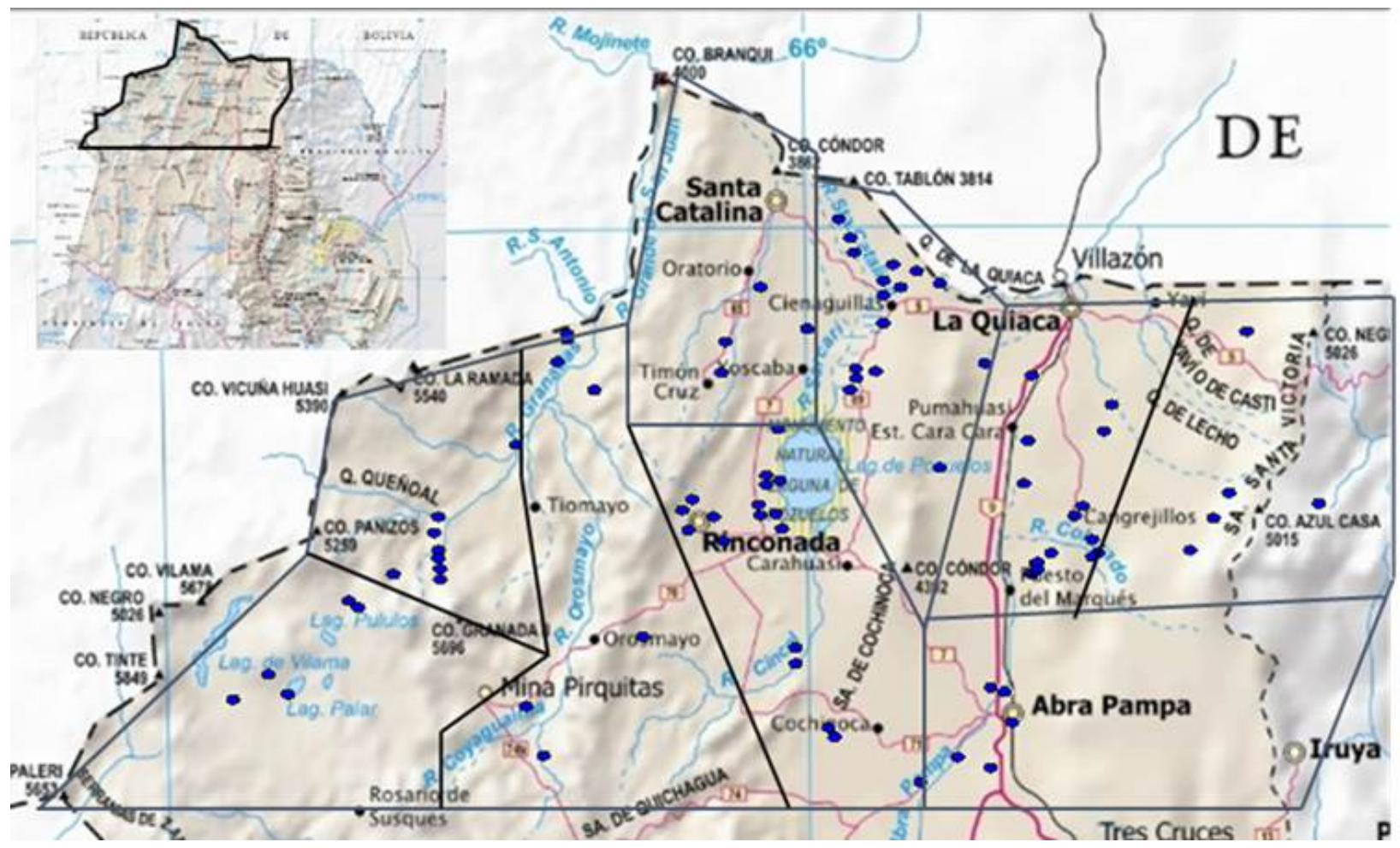

Figura 1. Distribución de tropas evaluadas en las cuencas de producción de la región relevada

Por otra parte, en el relevamiento original se basó en 6 caracteres etnozootécnicos (CEz) y 24 variantes fenotípicas (VFe) asociadas a: morfología (morfotipo y pigmentación de pezuñas, fenotipo de color (patrón pigmentario y diseño de la mancha blanca) y características de la fibra (pigmentación de mecha y tipo de mecha). $\mathrm{Y}$ se calcularon las respectivas frecuencias relativas (FRe, \%) por tropa. En el presente trabajo, se realizó la elección de las VFe que mejor explicaban la variabilidad y el proceso de estandarización de las poblaciones estudiadas en base a estudios previos (Hick, 2015; Hick et al., 2018a; 2018b). Las VFe se ilustran en la Figura 2 y las seleccionadas fueron: morfotipo calzado (MC), diseño de mancha total (M1), pigmentación de mecha crudo o ausente (PMCR) y tipos de mechas lustres (TML). Se utilizaron las respectivas FRe (\%) calculadas por tropa, donde según Renieri et al. (2009) y Hick (2015), aquellas con mayor presencia de animales con dichas $\mathrm{VFe}$, es decir mayor FRe (\%), será indicador de selección a favor de un biotipo determinado y por tanto de estandarización (menor primariedad o arcaísmo).

Luego se procedió a un análisis geoestadístico univariado de las cuatro $\mathrm{VFe}$ escogidas (MC, M1, PMCR y TML) mediante la utilización del paquete de estadística espacial del programa INFOSTAT (Di Rienzo et al., 2019). Como primer paso se realizó la transformación de las coordenadas geográficas originales de las tropas a coordenadas cartesianas (Xt e Yt) para faja o zona de ubicación geográfica 32719. Idéntica transformación se realizó para las coordenadas de los límites del área (Xlimi e Ylimi). A continuación, se utilizó el menú o la función automapa del paquete de estadística espacial de INFOSTAT 


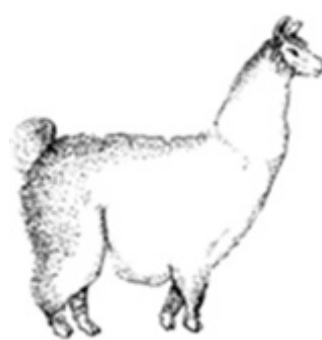

MC

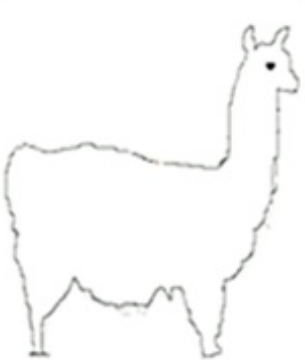

M1

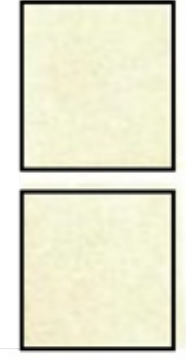

PMCR

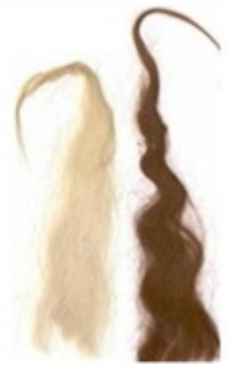

TML

MC: morfotipo calzado; M1: diseño de mancha total, PMCR: pigmentación de mecha crudo TML: tipos de mechas lustres Figura 2. Variantes fenotípicas (VFe) seleccionadas

(Córdoba, 2015) y a partir de los datos (FRe) y las coordenadas (Xt, Yt, Xlimi e Ylimi), se procedió de manera simultánea a: i) Obtener para cada VFe los respectivos semivariogramas empíricos y sobre éstos se ajustar distintos modelos de semivariograma teóricos (exponencial, esférico y gaussiano). Además, se obtuvieron de las distintas funciones semivariograma los parámetros rango (lag), la varianza estructural o sill parcial y la varianza nugget (efecto pepita). Para evaluar el grado de estructuración espacial para cada modelo se procedió a calcular la varianza estructural relativa $[\mathrm{RSV}=$ nugget $/$ (Sill parcial + nugget)]. Complementariamente se obtuvieron el criterio suma de cuadrados del error (SCE) y el criterio de información Akaike (AIC) para evaluar el grado de ajuste del modelo. ii) Realizar la

\section{Resultados y Discusión}

En primer lugar, en el Cuadro 1 se ilustran los valores de grado de estructuración, grados de ajuste y calidad de predicción de los diferentes modelos analizados y obtenidos del análisis geoestadístico univariado mediante la función automapa para las cuatro $\mathrm{VFe}$ seleccionadas. Los tres modelos de semivariograma teóricos (exponencial, esférico y gaussiano) denotan un comportamiento muy similar y a la vez dispar en cuanto a todos los valores. Por tanto, para estos casos no se descarta ningún modelo y la selección del modelo recae sobre aquel que posee los mayores de varianza estructural relativa (RSV) (Oliver and Webster, 2015; Córdoba. 2015). Por consiguiente, el modelo seleccionado fue el exponencial, ya que dicho modelo presentó para todos las VFe los mayores de varianza estructural relativa (RSV) resultando la estructuración espacial de mediana a muy alta (0.41 a 1.00). Luego la interpolación se realizó por kriging ordinario ya que no se observaron tendencias espaciales en ninguno de los ejes. A partir del modelo y parámetros del semivariograma exponencial, se obtuvieron los mapas de predicción de variabilidad interpolación en base a los parámetros de los semivariogramas ajustados, donde la función automapa de manera automática realiza la interpolación utilizando el método de kriging. El tipo de kriging varía según la existencia o no de tendencias espaciales que se analizan previamente. Por tanto, mediante la interpolación se obtuvieron los mapas de variabilidad espacial para las cuatro VFe. Finalmente, la interpolación mediante la función automapa también aportó los parámetros error medio (ME), error cuadrático medio (MSE), relación de las desviaciones cuadráticas medias (RMSE) y coeficiente de correlación de Pearson (rp). Dichos parámetros permiten analizar la calidad de predicción del modelo (Oliver and Webster, 2015).

espacial para las cuatro VFe seleccionadas e ilustrados en la Figura 3. Además se obtuvieron valores de predicción (rp) significativos en todos los casos (pvalor<0.0001) (Cuadro 1).

Los valores calculados y señalados para la varianza estructural relativa (RSV), indican que para las VFe seleccionadas las predicciones geoestadísticas serán más eficientes que aquellas obtenidas con métodos de predicción convencionales o sin tener en cuenta la información espacial. Por otra parte, los valores de predicción (rp) significativos confirman la existencia de una variación espacial de las variables estudiadas (VFe) con un buena capacidad de ser predichos (Oliver and Webster, 2015; Córdoba. 2015). Esto es un primer gran aporte del presente trabajo que permite optimizar y ser más preciso en la expresión de la variabilidad de las VFe estudiadas que la señalada en los primeros estudios para parte del área relevada por Frank et al. (1996), Frank et al. (1999) y luego para toda el área por Hick et al. (2009) y Hick et al. (2014). 


\begin{tabular}{|c|c|c|c|c|c|c|c|c|c|c|c|c|c|}
\hline \multirow[t]{2}{*}{$\begin{array}{l}\text { Variante } \\
\text { Fenotípica }\end{array}$} & \multirow[t]{2}{*}{ Modelo } & \multicolumn{5}{|c|}{ Estructuración } & \multicolumn{2}{|c|}{ Ajuste } & \multicolumn{5}{|c|}{ Predicción } \\
\hline & & Rango & $\begin{array}{c}\text { Sill } \\
\text { parcial }\end{array}$ & Nugget & Sill & RSV & SCE & AIC & $\overline{\mathrm{ME}}$ & MSE & RMSE & $\mathrm{r}_{\mathrm{p}}$ & p-valor \\
\hline \multirow[t]{3}{*}{$\mathrm{MC}$} & Exp & 26218.71 & 222.10 & 7.91 & 230.01 & 0.97 & 0.01 & -57.12 & -0.82 & 51.41 & 7.17 & 0.81 & $<0.0001$ \\
\hline & Sph & 37508.26 & 176.49 & 9.28 & 185.77 & 0.95 & 3.90E-03 & -60.55 & -0.85 & 51.68 & 7.19 & 0.81 & $<0.0001$ \\
\hline & Gau & 17141.00 & 173.91 & 18.39 & 192.30 & 0.90 & $2.70 \mathrm{E}-\mathrm{O} 3$ & 65.00 & -0.61 & 51.25 & 7.16 & 0.81 & $<0.0001$ \\
\hline \multirow[t]{3}{*}{ PMCR } & Exp & 11954.47 & 237.94 & 341.17 & 579.11 & 0.41 & 0.19 & -14.09 & -1.55 & 486.09 & 22.05 & 0.43 & $<0.0001$ \\
\hline & Sph & 13384.71 & 195.46 & 334.97 & 530.43 & 0.37 & 0.18 & -14.39 & -2.65 & 472.66 & 21.74 & 0.45 & $<0.0001$ \\
\hline & Gau & 8212.14 & 204.88 & 356.44 & 561.32 & 0.36 & 0.17 & -15.17 & -2.22 & 490.22 & 22.14 & 0.43 & $<0.0001$ \\
\hline \multirow[t]{3}{*}{ M1 } & Exp & 8116.29 & 386.65 & 0.00 & 386.65 & 1.00 & 0.04 & -35.35 & -0.93 & 238.18 & 15.43 & 0.60 & $<0.0001$ \\
\hline & Sph & 12271.50 & 340.04 & 0.00 & 340.04 & 1.00 & 0.03 & -39.13 & -1.16 & 217.75 & 14.76 & 0.63 & $<0.0001$ \\
\hline & Gau & 4309.84 & 331.50 & 0.00 & 331.50 & 1.00 & 0.01 & -49.33 & -1.36 & 213.33 & 14.61 & 0.65 & $<0.0001$ \\
\hline \multirow[t]{3}{*}{ TML } & Exp & 10938.83 & 339.36 & 93.76 & 433.12 & 0.78 & 0.03 & -36.55 & -0.65 & 211.68 & 14.55 & 0.64 & $<0.0001$ \\
\hline & Sph & 23323.73 & 313.59 & 105.75 & 419.34 & 0.75 & 0.03 & -37.30 & -0.80 & 205.49 & 14.33 & 0.65 & $<0.0001$ \\
\hline & Gau & 8113.12 & $267 \cdot 38$ & 123.18 & 390.56 & 0.68 & 0.03 & -35.15 & -0.84 & 215.96 & 14.70 & 0.63 & $<0.0001$ \\
\hline
\end{tabular}

Variante Fenotípica, MC: morfotipo calzado, M1: diseño de mancha total, PMCR: y TML pigmentación de mecha crudo: tipos de mechas lustres. Modelo, Exp, exponencial: Sph, esférico y Gau: gaussiano. RSV: varianza estructural relativa; SCE: suma de cuadrados del error; AIC: Criterio de información Akaike; ME: Error medio; MSE: error cuadrático medio; RMSE: relación de las desviaciones cuadráticas medias; $\mathrm{r}_{\mathrm{p}}$ : coeficiente de correlación de Pearson

Los cuatro mapas de variabilidad que se ilustran en la Figura 3 para cada VFe, muestran de manera clara una importante variación espacial, reflejado ello en los amplios rangos o recorridos de las FRe (\%), entre o \% y 80 \% según la VFe. En todos los mapas se observan áreas generalizadas con valores bajos de FRe (\%) de las VFe estudiadas indicando un estado de primariedad y por otra parte confirman la existencia de un área bien delimitada en la parte central y superior denominada como Cieneguillas (C) con valores elevados de FRe (\%). Por consiguiente, en dicha área se está registrando un proceso de estandarización, al menos incipiente, incrementando la frecuencia de individuos de gran cobertura, del tipo lustre y despigmentados. Ello determina que la VFe seleccionadas fueron las indicadas como ya lo había señalado Renieri et al. (2009) como criterios de selección para la población de Camélidos domésticos en general. Además, reflejaron los diferentes estados etnozootécnicos de la población de llamas estudiada en coincidencia con lo enunciado preliminarmente para pate del área por los primeros estudios de caracteres etnozootécnicos de Frank et al. (1996), Frank et al. (1999) y Hick et al. (2009) y con los estudios para la misma área y población realizados con índices de primariedad por parte de Hick (2015) y Hick et al. (2018a; 2018b).

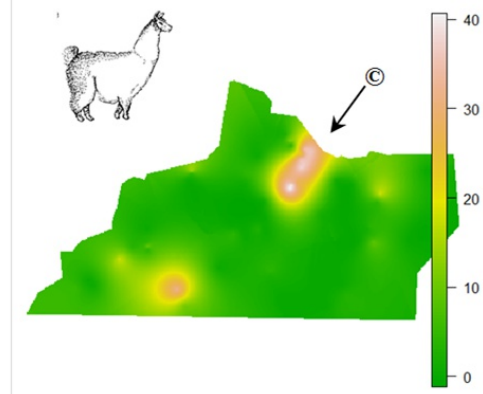

MC

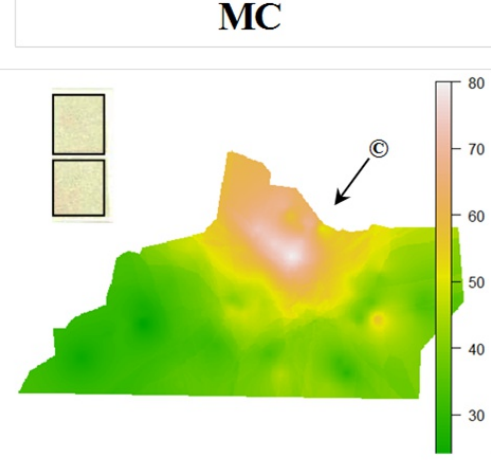

PMCR

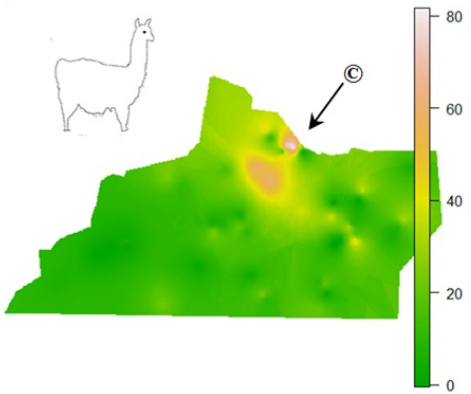

M1

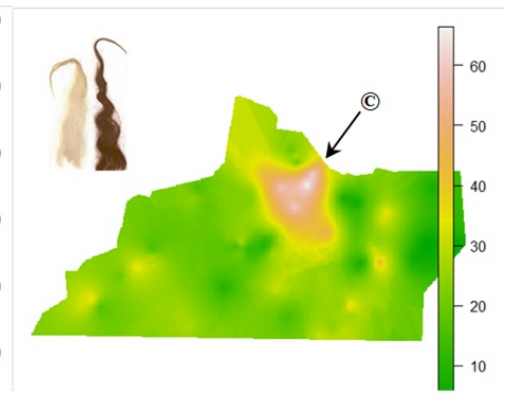

TML

MC: morfotipo calzado; M1: diseño de mancha total, PMCR: pigmentación de mecha crudo TML: tipos de mechas lustres. (C): Cieneguillas Figura 3. Mapas de predicción de variabilidad espacial de las VFe seleccionadas 
Finalmente, los mapas de variabilidad obtenidos mediante el análisis geoestadístico, señalan la existencia en el área Cieneguillas (C) de un "punto caliente" o isla de estandarización según el esquema geográfico de evolución de una población doméstica propuesto por Lauvergne (1996) y luego por Hick et al. (2019), donde se ha seleccionado a favor de las VFe elegidas y estudiadas en el presente trabajo. El tipo de animal o biotipo seleccionado es el observado en la Figura 4; no obstante, dicho biotipo no está presente en la totalidad de la tropa y en todas las del área como para hacer referencia a que la población del área Cieneguillas responde a un biotipo o raza estandarizado propiamente dicho o en el sentido clásico. Además, debería ser extensivo a otros caracteres etnozootécnicos (Hick et al., 2019).

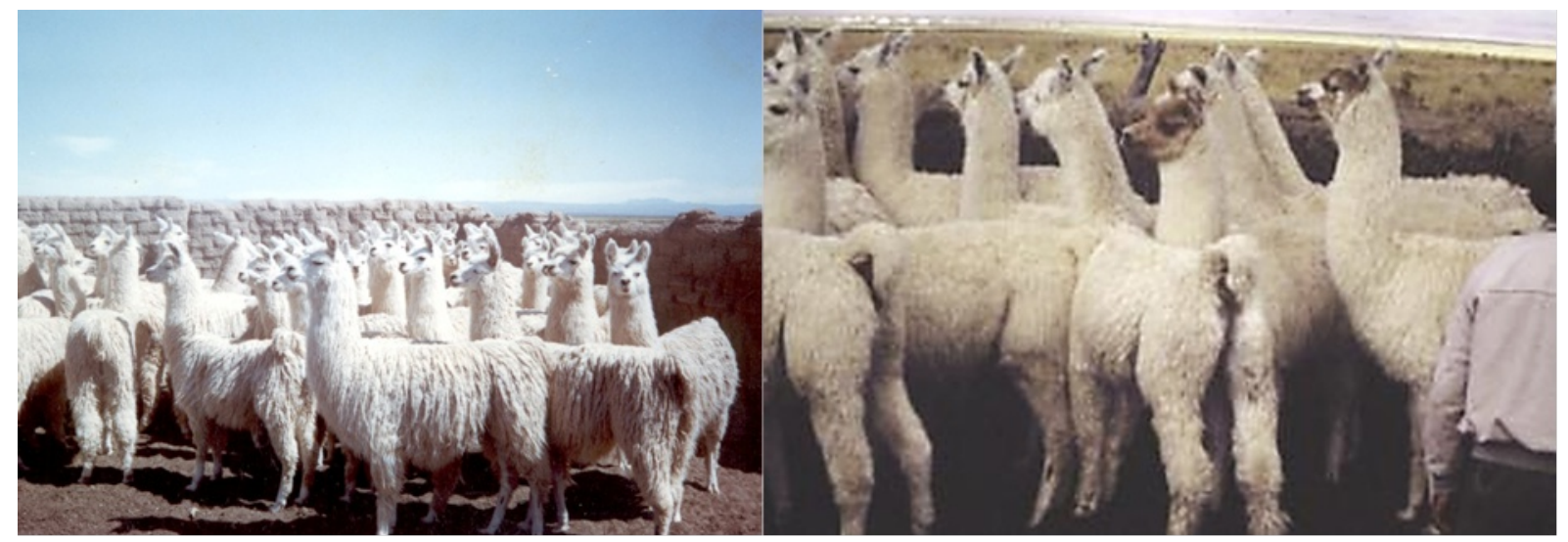

Figura 4. Tipo de animal o biotipo seleccionado en el área de Cieneguillas.

\section{Conclusiones}

Mediante el análisis geoestadístico univariado con la función automapa y el modelo exponencial, se pudo observar de manera eficiente y clara la importante variación espacial que presentan las tropas de llamas estudiadas del norte de la Provincia Jujuy. En base a las variantes fenotípicas escogidas, se pudo determinar y comprender mejor la variación espacial de la primariedad y estandarización presentada por dichas tropas de llama y enunciada en los antecedentes. El análisis realizado permitió delimitar diferentes áreas, donde si bien la mayor parte de la región relevada reviste una condición etnozootécnica de primariedad cuyas tropas de llamas están aún conformada por biotipos primitivos, en la región de Cieneguillas se observa un proceso de estandarización de las tropas con biotipos de aparición más reciente. Finalmente, la incorporación de más cantidad de tropas georreferenciadas, así como la utilización de técnicas geoestadísticas multivariadas y la incorporación de otros caracteres y variantes etnozootécnicas, podrían aún mejorar los resultados obtenidos en el presente trabajo.

\section{Literatura Citada}

Córdoba, M. 2015. Geoestadística. En: Balzarini, M.; Di Rienzo, J.; Tablada, M.; Gonzalez, L.; Bruno, C.; Córdoba, M.; Robledo, W. y Casanoves, F. Estadística y Biometría Ilustraciones del Uso de InfoStat en Problemas de Agronomía. Editorial Brujas, $\quad 2^{\circ} \quad$ Ed., 402 p. En: http://www.agro.unc.edu.ar/ mcia/archivos/Estadi stica \%20y \%20Biometria.pdf (Consulta 24/07/2020).

Di Rienzo J.A., Casanoves F., Balzarini M.G., Gonzalez L., Tablada M., Robledo C.W. InfoStat versión 2019. Centro de Transferencia InfoStat, FCA, Universidad Nacional de Córdoba, Argentina. En: http://www.infostat.com.ar (Consulta 24/07/2020).

FAO. 2012. Realización de encuestas y seguimiento de los recursos zoogenéticos. Directrices FAO: Producción y sanidad animal. No. 7. Roma. 17op. En: http://www.fao.org/3/baoo55s/baoo55soo.pdf (Consulta 24/07/2020).
Frank, E.N. 2001. Descripción y análisis de la segregación de fenotipos de color y tipos de vellón en llamas argentinas. Tesis de doctorado (UBA).

Frank, E.N.; Hick M.V.H.; Lamas H.E. y Vidal Castro, G. 1999. Estudio demográfico de las poblaciones de camélidos domésticos de las cuencas de Rinconada y Río Grande en la provincia de Jujuy, Argentina. En: I Cong. Latinoam. de Esp. en Peq. Rumiant. y Cam. Sud. Montevideo (RU).

Frank, E.N.; Hick, M.H.V.; Lamas, H.E. and Wehbe, V.E. 1996. A demographic study on comercial characteristics of fleece in Argentine Domestic Camelids (CAD) flocks. En: Gerken, M. \& Renieri, C. (eds.). Proc. of 2nd European Symp. on SAC. pp 5164.

Hick M.H.V., Castillo M.F. y Frank E.N. 2018a. Primariedad en tropas de llamas productoras de fibra del norte de la Provincia de Jujuy, Argentina. Actas Iberoamericanas de Conservación Animal 
(AICA), Vol. 12: 108-117. En: https://aicarevista.jimdo.com/n $\quad \% \mathrm{C}_{3}$ \%BAmeros/vol \%C3 \%BAmen-12-2018/ (Consulta 24/07/2020).

Hick M.H.V., Flores Gutiérrez, A., Castillo, M.F., Mamani-Cato, R.H. y Frank E.N. 2018b. Análisis geoestadístico de la primariedad en tropas de llamas productoras de fibra del norte de la Provincia de Jujuy, Argentina. VIII Congreso Mundial sobre Camélidos, 21 al 23 de noviembre Oruro, Bolivia. Memoria científica, Tomo II.: 128-129.

Hick, M.V.H. 2015. Caracterización etnozootécnica de poblaciones primarias (criollas) de ovinos, caprinos y Camélidos domésticos productores de fibra. Tesis doctoral. Universidad Católica de Córdoba. 207p. http://pa.bibdigital.uccor.edu.ar/137/1/TD_Hick.pd $\mathrm{f}$ (Consulta 24/07/2020).

Hick, M.V.H., Frank, E.N., Prieto A. y Castillo M.F. 2014. Etnozootecnia de poblaciones de Llamas (Lama glama) productoras de fibra de la Provincia de Jujuy, Argentina. Archivos Latinoamericanos de Producción Animal. 22 (1/2): 1-8. En: https://ojs.alpa.uy/index.php/ojs_files/article/view /2344/814 (Consulta 24/07/2020).

Hick, M.V.H.; Frank, E.N. y Mamani Cato, R.H. 2019. Demografía zootécnica aplicada a los Camélidos Sudamericanos Domésticos. Editorial Académica Española. 88 p. En: https://www.eaepublishing.com/catalog/index(Consulta 24/07/2020).

Hick, M.V.H.; Lamas, H.E.; Echenique, J.; Prieto, A.; Castillo, M.F. y Frank, E.N. 2009. Estudio demográfico de los atributos morfológicos y productivos en poblaciones de Llamas de la Provincia de Jujuy, Argentina. Animal Genetic Resources Information, Special Issue on Animal Natural Fibers, (45): 71-78. En: http://www.fao.org/3/i1102t/i1102to4.pdf (Consulta 24/07/2020).
INDEC. 2002. Censo Nacional Agropecuario 2002. En: http://www.indec.gov.ar/agropecuario/cna.asp (Consulta 11/02/2014).

Lauvergne, J.J. 1996. Clasificación de los recursos genéticos de los mamíferos domésticos con extensión a los Camélidos Sudamericanos. Actas $1^{\mathrm{O}}$ Seminario Internacional de Camélidos Sudamericanos domésticos. Frank, E.N and C. Renieri (Eds): 59-67. En: http://pa.bibdigital.uccor.edu.ar/id/eprint/1459 (Consulta 24/07/2020).

Frank, E.N. 2001. Descripción y análisis de la segregación de fenotipos de color y tipos de vellón en llamas argentinas. Tesis de doctorado (UBA).

Oliver, M.A and Webster R. 2015. Basic steps in geostatistics: the variogram and kriging. Springer, New York. 10op.

Renieri, C; Frank, E.N.; Rosati, A.Y. y Antonini, M. 2009. Definición de razas en llamas y alpacas. Animal Genetic Resources Information, Special Issue on Animal Natural Fibers, (45): 45-54. En: http://www.fao.org/tempref/docrep/fao/o12/i1102t /i1102to3.pdf (Consulta 24/07/2020).

SENASA. 2012. Indicadores ganaderos. En: http://www.senasa.gov.ar/indicadores.php?in=1 (consulta 29/10/2012).

Tixier-Boichard, M.; Ayalew, W. and Jianlin, H. 2007. Inventory, characterization and monitoring. Animal Genetic Resources Information, 42: 29-47. En: http://www.fao.org/3/ioo72t/ioo72to3.pdf (Consulta 24/07/2020). 